\title{
HIPERTENSION INTRACRANEAL IDIOPATICA, CASO CLINICO: HALLAZGOS EN RM Y REVISION DE LA LITERATURA
}

Drs. Paloma García I, Francisco Javier De Castro G, José Francisco Asensio C, Juan Carlos Paniagua E, Andrés Framiñán de Miguel.

Servicio de Radiodiagnóstico. Sección Neurorradiología. Hospital Universitario. Salamanca. España.

\begin{abstract}
Idiopathic intracranial hypertension is charactericed by increased intracranial pressure in the absense of a space occupying lesion or obstruction to the circulation of cerebroespinal fluid (CSF). Usually self-limited, but often relapsing. Diagnosis is based on a record of intracranial pressure of over a limit of $250 \mathrm{mmH} 2 \mathrm{O}$. Bilateral papilloedema and paresia of the VI cranial nerve is seen in most patients. Morbidity is basically due to possible loss of vision associated with atrophy of the optic nerve. Management include administration of acetazolamide, repeated lumbar punctures and weight loss. Magnetic resonance (MR) imaging demostrated "empty sella", subarachnoid spaces are enlarged, distension of the perioptic subarachnoid space and enhancement of the prelaminar optic nerve Key words: Benign intracranial hypertension. Idiopathic intracranial hypertension, Pseudotumor cerebri, Magnetic resonance.
\end{abstract}

Resumen: La hipertensión intracraneal idiopática es un síndrome neurológico caracterizado por síntomas y signos de hipertensión intracraneal en ausencia de lesión estructural o de hidrocefalia. En general, autolimitada, aunque con frecuencia recidiva. El diagnóstico se basa en la medición de la presión del líquido cefalorraquídeo que se eleva por encima de $250 \mathrm{~mm}$ de $\mathrm{H}_{2} \mathrm{O}$. Normalmente los resultados del examen neurológico son normales excepto por la aparición de papiledema y posible afectación del VI par. La principal complicación es la pérdida visual

García P. Hipertensión intracraneal idiopatía, caso clínico: Hallazgos en RM y revisión de la literatura. Rev Chil Radiol 2005; 11: 138-141.

Correspondencia: Dra. Paloma García I.

Paseo San Vicente 58-182. Fono: 609973828.

E-mail:palomeil@hotmail.com que puede ser irreversible. El tratamiento se basa en la repetición de punciones lumbares evacuadoras combinado con el uso de diuréticos y dieta hipocalórica. Con la aparición de la resonancia magnética se han descrito varios signos asociados a esta enfermedad como son la disminución del tamaño ventricular, el incremento del espacio subaracnoideo, la presencia de silla turca vacía, distensión del espacio perióptico y realce tras contraste de los nervios ópticos.

Palabras clave: Hipertensión intracraneal benigna, Hipertensión intracraneal idiopática, Pseudotumor cerebral, Resonancia magnética.

\section{Introducción}

La hipertensión intracraneal idiopática $(\mathrm{HICl})$ es un síndrome neurológico caracterizado por síntomas y signos de hipertensión intracraneal sin evidencia de lesión estructural o de hidrocefalia ${ }^{(1)}$. Suele ser autolimitada aunque con tendencia a la recidiva $^{(2)}$. Es más frecuente en mujeres de edad media, obesas ${ }^{(1,3)}$ y/o con irregularidades menstruales. La manifestación clínica más frecuente es la cefalea asociada a síntomas oculares tales como disminución de la agudeza visual, escotomas y fotopsias así como diplopía por afectación característica del VI par(2). Incluso puede llegar a provocar ceguera irreversible. La elevación de la presión del líquido cefaloraquídeo (LCR) por encima de $250 \mathrm{~mm}$ de $\mathrm{H}_{2} \mathrm{O}$, tras descartar patología intracraneal es diagnóstica de la enfermedad ${ }^{(4)}$. Esta situación mantenida, provoca la aparición de signos en las imágenes de resonancia magnética (RM) que permite excluir otras patologías con presentaciones clínicas similares ${ }^{(5,6)}$.

\section{Caso Clínico}

Mujer de 62 años, hipertensa bien controlada con dieta y tratamiento diurético con tiazida que 
acude a la consulta de oftalmología por presentar lagrimeo, visión borrosa y diplopía en ojo derecho desde hace dos meses. No aquejaba cefalea ni fiebre. Las cifras de tensión arterial eran normales. Refería un episodio similar hacía 30 años, motivo por el que había sido ingresada y diagnosticada de pseudotumor cerebri. Fue tratada con corticoides y punciones lumbares permaneciendo asintomática y no había seguido controles posteriores. Exploración física: hábito picnico (IMC $27,1 \mathrm{~kg} / \mathrm{m}^{2}$ ), exploración neurológica y oftalmológica normal salvo por la presencia de papiledema bilateral y cuadrantanopsia inferior nasal derecha. Los resultados de los estudios sistemáticos de sangre y orina se encontraban dentro de los límites de la normalidad. Valorada por el Servicio de Neurocirugía se decide la solicitud de una RM cerebral para valorar lesiones en el quiasma óptico. La exploración se realizó en un equipo Signa Horizon 1,5 T (General Electric, Milwaukee, Wisconsin). El protocolo incluyó secuencias sagital espín eco potenciadas en T1 (TR: 500; TE: 20), axial eco espín rápidas potenciadas en densidad protónica (TR: 2000; TE: 28), axial y coronal eco espín rápidas potenciadas en T2 (TR: 4000; TE: 120) y coronal STIR (TR: 3200; TE: 18; TI: 160). Tras la administración de gadolinio intravenoso se obtuvieron secuencias coronales potenciadas en T1 (TR: 600; TE: 15). Se observó una distensión del espacio subaracnoideo perióptico de forma bilateral (Figura 1), la presencia de una silla turca parcialmente vacía (Figura 2) y aumento de ambas cisternas trigeminales con remodelación ósea del cavum de Meckel (Figura 3).

Ante estos hallazgos, se realizó una punción lumbar dando salida a un LCR claro con presión de apertura de $260 \mathrm{~mm} \mathrm{H}_{2} \mathrm{O}$ y estudio cito-bioquímico normal.

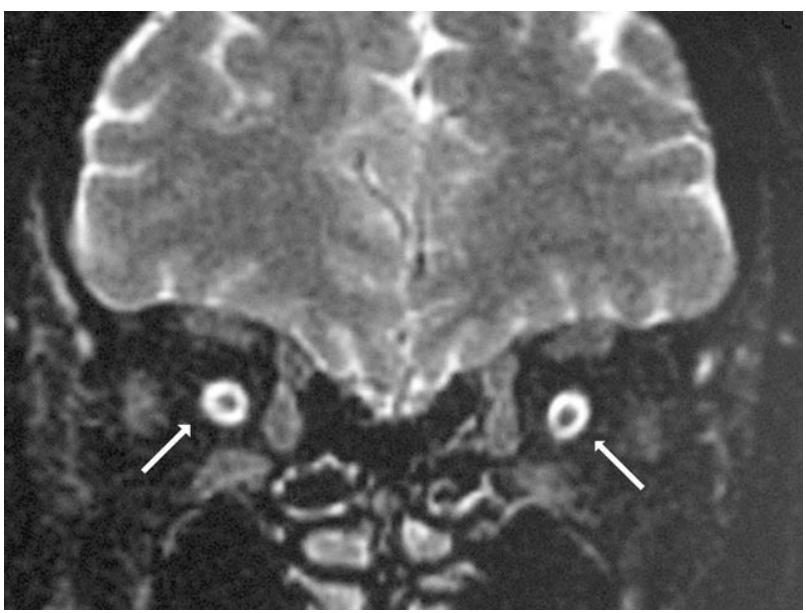

Figura 1. Imagen de RM coronal en secuencia STIR que muestra una distensión del espacio subaracnoideo perióptico bilateral (flechas).

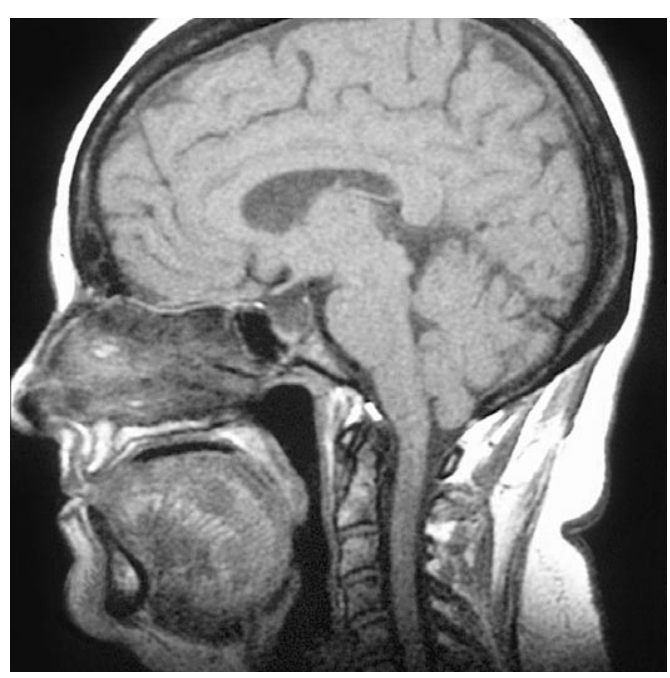

Figura 2. RM sagital ponderada en T1 donde se aprecia concavidad de la glándula pituitaria y desplazamiento posterior del infundíbulo con resto de silla turca rellena de LCR.
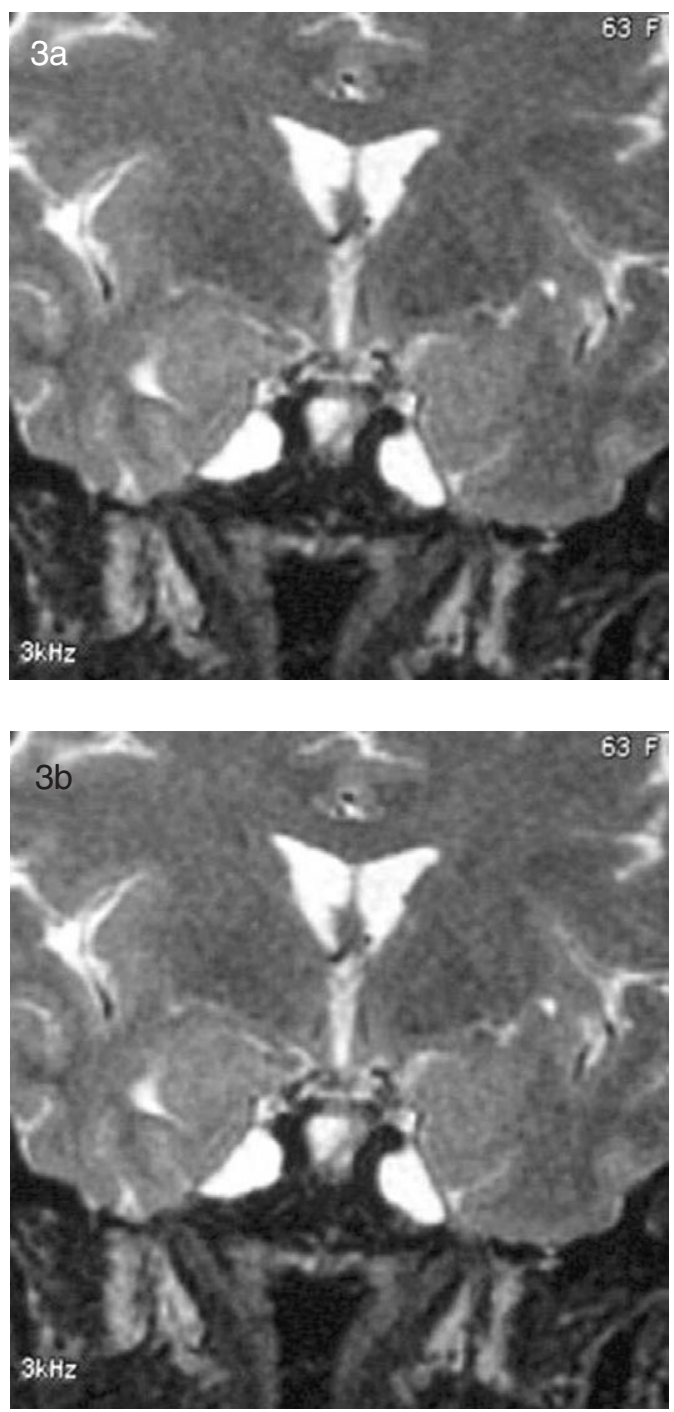

Figura 3 a, b. Imágenes de RM en incidencias axial (a). coronal (b) ponderadas en T2 que muestran un ensanchamiento de las cisternas trigeminales con remodelación ósea del cavum de Meckel. 


\section{Discusión}

El diagnóstico de $\mathrm{HICl}$ se basaba en los criterios de Dandy modificados ${ }^{(7,8)}$. Sin embargo, dados los avances en las técnicas de neuroimagen se han propuesto nuevos criterios diagnósticos (Tabla I) ${ }^{(9)}$. Los valores normales de apertura de la presión del LCR se encuentran entre $70-200 \mathrm{~mm} \mathrm{H}_{2} \mathrm{O}^{(10)}$. Se acepta como criterio diagnóstico una presión superior a $250 \mathrm{~mm} \mathrm{H}_{2} \mathrm{O}$ teniendo en cuenta que la presión del LCR fluctúa por lo que en ocasiones debe repetirse la punción. Hasta en el $90 \%$ de los casos son mujeres con una edad media de 31 años ${ }^{(7)}$. La $\mathrm{HICl}$ se considera una entidad de etiología desconocida. Sin embargo, se han admitido varias teorías sobre su patogénesis incluyendo edema cerebral(11), descenso de la absorción ${ }^{(12)}$ de LCR por las vellosidades aracnoideas, aumento en la producción de $\operatorname{LCR}^{(2)}$, aumento del volumen intravascular ${ }^{(13)}$ y aumento de la presión venosa intracraneal o trombosis ${ }^{(14)}$. Estas teorías traducen una alteración en la funcionalidad de los senos venosos que condiciona un trastorno en el drenaje de LCR.

Aunque entre las posibles causas se han propuesto alteraciones endocrinológicas (hipertiroidismo, hipotiroidismo, hipoparatiroidismo, insuficiencia suprarrenal, síndrome de Cushing) $)^{(15)}$. Solamente la obesidad y el incremento de peso reciente se han relacionado estadísticamente con esta afección ${ }^{(16)}$.

Una de las principales características de esta entidad es su gran variabilidad en cuanto a su sintomatología. Hay enfermos con una cefalea incapacitante, mientras que en otros casos cursa de forma asintomática y sólo se aprecia la reducción del campo visual como ocurría en nuestro caso. Santos y cols. ${ }^{(17)}$ describen cuatro formas de presentación: aparición de síntomas de hipertensión intracraneal: cefalea, náuseas, vómitos, diplopía; instauración aislada de síntomas visuales: oscurecimientos visuales fugaces, visión borrosa, disminución de la agudeza visual; coexistencia de síntomas de hipertensión intracraneal y visuales, y por último, descubrimiento de un papiledema aislado en un examen oftalmológico de rutina.

Aunque la cefalea es el síntoma más frecuente no siempre aparece. Se han descrito diplopía, oscuraciones visuales transitorias, pérdida de visión progresiva o brusca, y más raramente escotomas centelleantes y dolor retroocular ${ }^{(18)}$. En la exploración neurológica, el hallazgo más característico es la presencia de papiledema $(98-100 \%)^{(17)}$ que en menos del $10 \%$ es unilateral ${ }^{(18)}$. En ocasiones, entre el $10 \%$ y $40 \%$ de los casos, se aprecia paresia del VI par ${ }^{(10)}$.

La dieta hipocalórica junto con la práctica de punciones lumbares repetidas, una o dos veces por semana, es el primer tratamiento a considerar ${ }^{(3)}$. Este
Tabla I. Criterios diagnósticos de $\mathrm{HICl}$

1. Si existen síntomas, éstos se deben a la hipertensión intracraneal o al papiledema.

2. Si existen signos, éstos se deben a la hipertensión intracraneal o al papiledema.

3. La elevación de la presión intracraneal debe ser medida en decúbito lateral.

4. La composición del LCR debe ser normal.

5. No existen evidencias de hidrocefalia, masa estructural o lesión vascular en RM o TC con contraste, en los pacientes habituales, o en RM con estudio venoso en el resto de los pacientes.

6. No se identifican otras causas de hipertensión intracraneal.

método consigue, por sí mismo, remisiones transitorias ${ }^{(3)}$. Otras medidas terapéuticas incluyen el uso de diuréticos como la acetazolamida. En caso de no respuesta a estos tratamientos, puede ser necesaria la derivación lumboperitoneal o la fenestración del nervio óptico(19).

La elevación de la presión del LCR se traduce en el estudio con RM en una distensión del espacio subaracnoideo perióptico en el $45 \%$ de los pacientes con abalonamiento de la papila, que protuye en el aspecto posterior del globo ocular(5). Asimismo, el efecto prolongado de pulsos de LCR debido a la alta presión puede conducir a una herniación de un aracnocele a través de un defecto en el diafragma selar, provocando concavidad de la glándula pituitaria ${ }^{(20)}$ e incluso en fases muy evolucionadas silla turca vacía. Otros signos descritos son la tortuosidad y realce tras contraste de los nervios ópticos. En un estudio reciente se usaron técnicas de difusión, descartando la existencia de edema cerebral como causa de la $\mathrm{HICl}^{(21)}$.

\section{Conclusión}

Las imágenes de RM de los nervios ópticos y de la glándula pituitaria pueden proporcionar claves importantes para el diagnóstico de la $\mathrm{HICl}$ particularmente cuando los hallazgos clínicos son equívocos. En nuestra paciente, probablemente debido a la cronicidad del proceso, la clínica predominante era la visual y se había producido el remodelamiento del cavum de Meckel asociado al resto de hallazgos típicos. 


\section{Bibliografía}

1. Ahlskog JE, O’Neill BP. Pseudotumor cerebri. Ann Intern Med 1982; 97: 249-256.

2. Salman MS, Kirkham FJ, MacGregor DL. Idiopathic «benign» intracranial hypertension: case series and review. J Child Neurol 2001; 16: 465-70.

3. Weisberg LA. Benign intracranial hypertension. Medicine (Baltimore) 1975; 54: 197-207.

4. Corbett JJ, Metha MP. Cerebroespinal fluid pressure in normal obese subjects and patients with pseudotumor cerebri. Neurology 1983; 33: 1386-8.

5. Brodsky MC, Vaphiades M. Magnetic resonance imaging in pseudotumor cerebri. Ophthalmology 1998; 105: 1686-1693.

6. Silbergleit R, Junck L, Gebarski SS, Hatfield MK. Idiopathic intracranial hypertension (pseudotumor cerebri): MR imaging. Radiology 1989; 170: 207-209.

7. Wall M, George D. Idiopathic intracranial hipertension. A prospective study of 50 patients. Brain 1991; 114: 155-180.

8. Smith JL. Whence pseudotumor cerebri? J Clin Neuroophthalmol 1985; 5: 55-56.

9. Friedman D, Jacobson DM. Diagnostic criteria for idiopathic intracranial hypertension. Neurology 2002; 59: 1492-5.

10. Fishman RA. Disorders of intracraneal pressure: hydrocephalus, brain edema, pseudotumor, intracraneal hypotension, and related disorders. In: Cerebrospinal fluid in diseases of the nervous system. $2^{\text {nd }}$ ed. Philadephia: WB Saunders. 1992: 103-55.

11. Sahs AL, Joynt RJ. Brain swelling of unknown cause. Neurology 1956; 6: 791-803.

12. Raichle ME, Grubb RL Jr, Phelps ME, et al. Cerebral hemodynamics and metabolism in pseudotumor cerebri. Ann Neurol 1978; 4: 104-11.
13. Rekate HL, Brodkey JA, Chizeck HJ, et al. Ventricular volume regulation: a mathematical model and computer simulation. Pediatr Neurosci 1988; 14: 7784.

14. Junck L. Benign intracranial hypertension and normal pressure hydrocephalus: theoretical considerations. In: Miller TD, Teasdale GM, Rowan JO, Galbraith SL, Mendelson AD, ed. Intracranial pressure VI. Berlin: Springer, 1986; 447-450.

15. Fishman RA. The pathophysiology of pseudotumor cerebri: an unsolved puzzle. Arch Neurol 1984; 41: 257-8.

16. Giuseffi V, Wall M, Siegel PZ, Rojas PB. Symptoms and disease associations in idiopathic intracraneal hypertension (pseudotumor cerebri): a case-control study. Neurology 1991; 41: 239-44.

17. Santos S, López del Val LJ, Pascual LF y col. Pseudotumor cerebral: análisis de nuestra casuística y revisión de la literatura. Rev Neurol 2001; 33: 1106-11.

18. Corbett JJ, Savino PJ, Thompson HS et al. Visual loss in pseudotumor cerebri. Arch Neurol 1982; 39: 461-74.

19. Kelman SE, Heaps R, Wolf A, Elman MJ. Optic nerve decompression surgery improves visual function in patients with pseudotumor cerebri. Neurosurgery 1992; 30: 391-5.

20. Yuh W, Zhu M, Taoka T, Quets JP, Maley JE, Muhonen $M G$ et al. MR imaging of pituitary morphology in idiopathic intracranial hypertension. J Magn Reson Imaging 2000; 12: 808-13.

21. Bastin ME, Sinha S, Farrall A, Wardlaw JM, Whittle IR. Diffuse brain oedema in idiopathic intracranial hypertension: a quantitative magnetic resonance imaging study. J Neurol Neurosurg Psychiatry 2003; 74: 1693-1696. 